The Free Internet Journal for Organic Chemistry
Paper

Arkivoc 2019, part v, 0-0

to be inserted by editorial office

\title{
Synthesis of poly-functionalized pyrazoles under Vilsmeier-Haack reaction conditions
}

\section{Aleksandr V. Popov, ${ }^{*}$ Valentina A. Kobelevskaya, Ludmila I. Larina, and Igor B. Rozentsveig}

A. E. Favorsky Irkutsk Institute of Chemistry, Siberian Branch of the Russian Academy

of Sciences, Irkutsk, 664033, Russia

E-mail:popov@irioch.irk.ru

With thanks to Professor Galina G. Levkovskaya for her great scientific interest

Received 03-17-2019

Accepted 06-06-2019

Published on line $07-03-2019$

\section{Abstract}

Synthesis of 1,3-disubstituted 5-chloro- $1 \mathrm{H}$-pyrazole-4-carbaldehydes was achieved by formylation of the corresponding 5-chloro-1H-pyrazoles under Vilsmeier-Haack conditions.
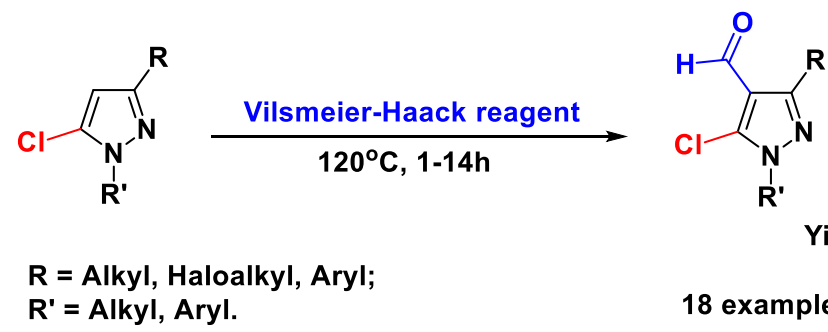

Yields: $4-67 \%$

18 examples

Keywords: Pyrazoles, 5-chloropyrazoles, pyrazole-4-carbaldehydes, Vilsmeier-Haack reaction, formylation 


\section{Introduction}

The pyrazole core is a privileged structural motif of the modern drugs and bioactive natural compounds. This heterocycle and its derivatives are important building blocks for organic, bioorganic, pharmaceutical and supramolecular chemistry as well as materials science. ${ }^{1-9}$ In fact, the pyrazole ring ranks $44^{\text {th }}$ in a frequency among 351 ring systems found in currently marketed drugs. ${ }^{10}$ The incorporation of functional groups into the pyrazolic architecture attracts considerable interest because this modification opens the possibility for further transformations. These poly-functionalized heterocyclic systems have shown promising applications in the search of new biologically active candidates for drug discovery as well as polydental ligand systems. Among them the pyrazoles bearing a halogen atom and formyl group are of particular interest. ${ }^{11}$

Thus, halopyrazoles show a diverse range of biological activity. ${ }^{12}$ Moreover, the introduction of a halogen atom into the pyrazole ring represents often a first step in the synthesis of hard-to-reach derivatives. Many of the metal-catalyzed carbon-carbon coupling reactions, such as Sonogashira, Heck-Mizoroki, Stille, Negishi, and Suzuki-Miyaura are based on halopyrazoles. ${ }^{13-16}$ On the other hand, 4-formylpyrazoles are currently used for the preparation of nitronylnitroxyl pyrazole-containing ligands, which are promising building blocks in design of new heterospin magnetics, ${ }^{17}$ as well as diverse heteropolycyclic derivatives. ${ }^{18}$ It is worth mentioning that the poly-condensed heterocyclic ring system, which was synthesized from 5-chloro- $1 \mathrm{H}$-pyrazole-4carbaldehyde, exhibited potent anti-inflammatory and analgesic activities. In this case, the incorporation of pyrazole nucleus to quinoxaline moiety caused significant biologically activities. ${ }^{19}$

Generally, the methods for synthesis of formyl(halo)pyrazoles can be divided into two groups. The first approach presupposes the use of polyfunctional starting materials in cascade assembly of the heterocyclic core. The second one is based on the incorporation of the functional group into previously prepared pyrazoles. The modification of the existing heterocyclic system seems to be a more convenient approach to target compounds.

The methods for preparation of halo-substituted formyl pyrazoles, to the best of our knowledge, have rarely been reported. One of the approaches to 3/5-chloro (or 5-bromo)-4-formyl pyrazole derivatives is based on pyrazolones which undergo haloformylation under Vilsmeier-Haack reaction. 13,20,21 Taking into account that pyrazolones are produced by refluxing of hydrazines with 1,3-dicarbonyl compounds, ${ }^{22}$ this method was extended only for derivatives obtained from the most available representatives of 1,3 -diketones or $\beta$ ketoesters. ${ }^{13}$ In addition, the pyrazolones were often obtained in low yields. ${ }^{23,24}$

Other pathways to target compounds are based on the reaction of 3-halo- $1 \mathrm{H}$-pyrazoles with organometallics ${ }^{25}$ or diazonium derivatives. ${ }^{26}$ However, low availability of the starting materials as well as high laboriousness are disadvantages of these approaches. Thus, a straightforward approach to functionalized pyrazoles from available initial reagents is of interest. Herein, we report a general and efficient method for the synthesis of halo-substituted formylpyrazoles by simple formylation of corresponding halopyrazoles.

\section{Results and Discussion}

Our aim was to formylate 1,3-disubstituted 5-chloro- $1 H$-pyrazoles 1 under Vilsmeier-Haack conditions to yield the corresponding 5-chloro- $1 \mathrm{H}$-pyrazole-4-carbaldehydes 2 (Scheme 1). Most of the starting materials were readily prepared by base-catalyzed condensation of dichlorovinyl ketones with hydrazines (Scheme 2). ${ }^{27-30}$ An important advantage of this method is highly selective formation of 5-chloropyrazoles. 

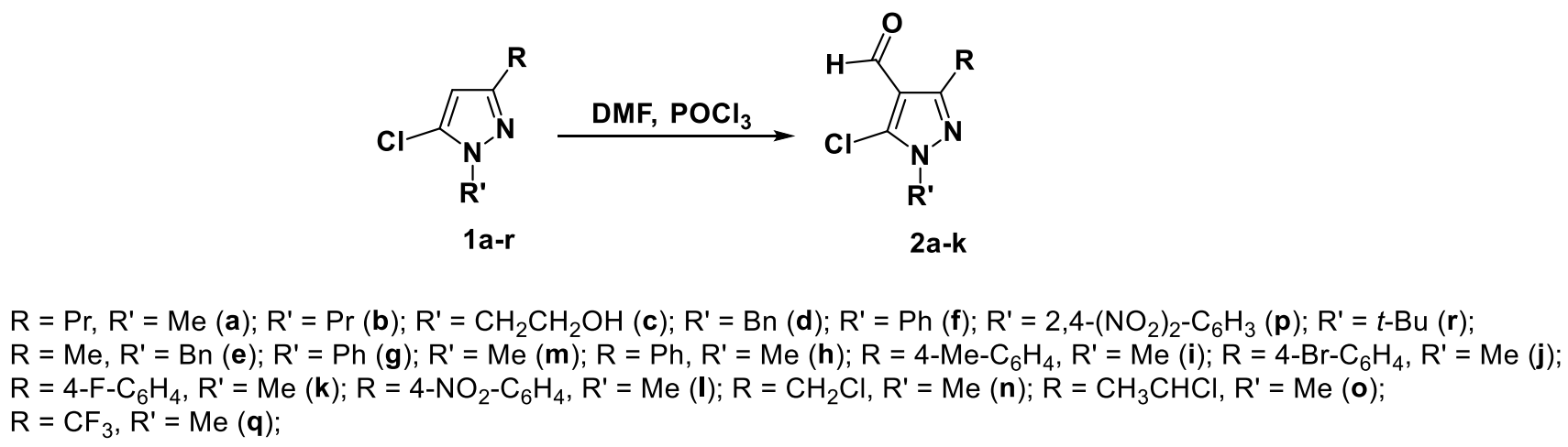

Scheme 1. The general approach for preparation of 5-chloro- $1 \mathrm{H}$-pyrazole-4-carbaldehydes.

Moreover, since dichlorovinyl ketones can be obtained from readily available reagents (vinylidene chloride and acyl chlorides $\left.{ }^{31-34}\right)$, synthesis of a series of 3-substituted pyrazoles 1 a priori could be easily accessible.
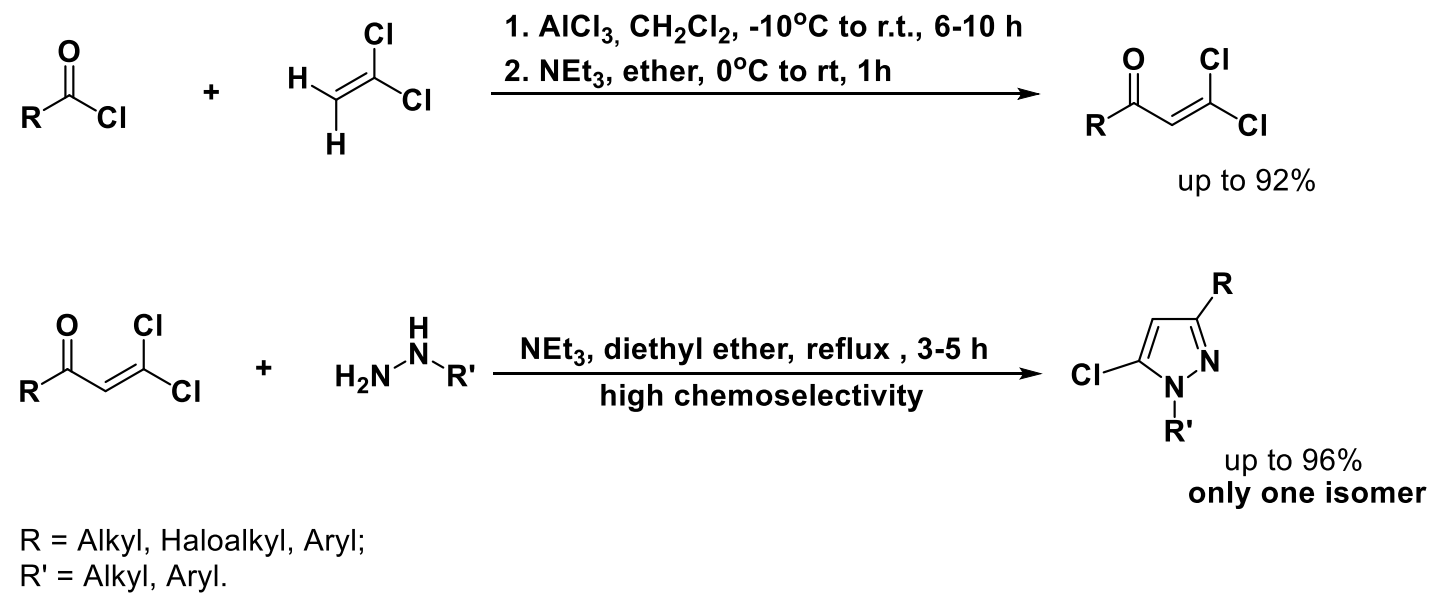

Scheme 2. Simple and efficient method for preparation of 5-chloro- $1 H$-pyrazoles.

First, we sought the optimal reaction conditions using 1-methyl-3-propyl-5-chloro-1H-pyrazole $(1 \mathrm{a} ; \mathrm{R}=$ $\operatorname{Pr}, R^{\prime}=\mathrm{Me}$ ) (Sheme 1) as a model compound and the results are shown (Table 1). When reaction was carried out at $70^{\circ} \mathrm{C}$, no products were observed at all (Entry 1 ). The best results were obtained when the pyrazole 1a was treated with excess of DMF and $\mathrm{POCl}_{3}$ at $120^{\circ} \mathrm{C}$. For instance, using a 5 -fold excess of DMF and 2-fold excess of $\mathrm{POCl}_{3}$ at $120^{\circ} \mathrm{C}$ for $2 \mathrm{~h}$ gives carbaldehyde $2 \mathrm{a}$ in $55 \%$ yield instead of $32 \%$ only for 2 -fold excess of the same reagents (Entries 2, 3).

The use of excess of $\mathrm{POCl}_{3}$ leads to increasing amounts of chloroiminium ions, the key intermediates of the Vilsmeier-Haack reaction, and therefore favors the formation of target pyrazole. The excess of DMF ensures homogeneity of the process and solvates the released hydrogen chloride. The yield of $\mathbf{2 a}$ is not increased when more than 6 and 4 equivalents of $\mathrm{DMF}$ and $\mathrm{POCl}_{3}$ are used. At this ratio of reagents, a further increase in the reaction time does not affect the yield of the target product (Entries 7,8 ). The use of microwave assistance did not favor the formation of target pyrazole 2 a (Entries 4,5$)$. Thus, the optimum conditions of the process are heating the mixture of 5-chloropyrazole 1a with 6 equivalents of DMF and 4 equivalents of $\mathrm{POCl}_{3}$ at $120^{\circ} \mathrm{C}$ for $1 \mathrm{~h}$. Under such conditions, yield of the target $1 \mathrm{H}$-pyrazole-4-carbaldehyde $2 a$ reached $67 \%$ (Entry 7 ). 
Table 1. Optimization of the reaction conditions (ratio of the 3 reactants, temperature, duration of reaction) for the conversion of 1-methyl-3-propyl-5-chloro-1H-pyrazole $\left(1 \mathrm{a} ; \mathrm{R}=\mathrm{Pr}, \mathrm{R}^{\prime}=\mathrm{Me}\right)$ into 1-methyl-3-propyl-5chloro-1H-pyrazole-4-carbaldehyde $\left(2 \mathrm{a} ; \mathrm{R}=\mathrm{Pr}, \mathrm{R}^{\prime}=\mathrm{Me}\right)$ (Scheme 1)

\begin{tabular}{cccccc}
\hline Entry & $\begin{array}{c}\text { Molar ratio } \\
\text { 1a }: \text { DMF }: \mathrm{POCl}_{3}\end{array}$ & $\mathrm{~T},{ }^{\circ} \mathrm{C}$ & Time, $\mathrm{h}$ & $\begin{array}{c}\text { Conversion } \\
\text { of 1a, \% }\end{array}$ & $\begin{array}{c}\text { Yield }{ }^{\mathrm{b}} \text { of } \\
\mathbf{2 a}, \%\end{array}$ \\
\hline 1 & $1: 2: 2$ & 70 & 7 & 4 & trace \\
2 & $1: 2: 2$ & 120 & 7 & 51 & 32 \\
3 & $1: 5: 2$ & 120 & 2 & 74 & 55 \\
$4^{\mathrm{c}}$ & $1: 5: 2$ & 120 & 1 & 62 & 45 \\
$5^{\mathrm{c}}$ & $1: 5: 2$ & 140 & 2 & 9 & trace \\
6 & $1: 5: 3$ & 120 & 2 & 82 & 61 \\
7 & $1: 6: 4$ & 120 & 1 & 92 & 67 \\
8 & $1: 6: 4$ & 120 & 2 & 95 & 65 \\
9 & $1: 6: 4$ & 140 & 1 & 89 & 66 \\
10 & $1: 6: 5$ & 120 & 2 & 87 & 64 \\
\hline
\end{tabular}

a $2 \mathrm{mmol}$ of 1a were used in screening of the reaction conditions.

${ }^{b}$ Yield of the isolated product.

c Under microwave activation

Under the selected optimized conditions, the various 5 -chloropyrazoles $\mathbf{1 b}$-r were examined (Table 2). The experiments suggested that the reaction result is dependent on the substrate structure. To our delight, like model compound $\mathbf{1 a}$, a total conversion of starting 5-chloro-1,3-dialkyl-substituted pyrazoles $\mathbf{1} \mathbf{b}, \mathbf{d}, \mathbf{e}, \mathbf{m}, \mathbf{n}$ (Entries $2,4,5,13,14)$ was achieved after refluxing at $120^{\circ} \mathrm{C}$ for $1-2.5 \mathrm{~h}$. In contrast, 1-aryl and 3-aryl substituted pyrazoles 1f-1l needed much more time to complete the reaction (Entries 6-12). 
Table 2. Preparation of 1,3-disubstituted 5-chloro-1H-pyrazole-4-carbaldehydes 2 by formylation of the corresponding 5-chloro-1H-pyrazoles 1 under Vilsmeier-Haack conditions (Scheme 1) ${ }^{a}$

\begin{tabular}{|c|c|c|c|c|c|}
\hline Entry & $\begin{array}{c}\text { Compound } \\
\text { number }\end{array}$ & $\mathrm{R}$ & $\mathrm{R}^{\prime}$ & Time (h) ${ }^{b}$ & Yield, \% \\
\hline 1 & $2 a$ & $\operatorname{Pr}$ & Me & 1 & 67 \\
\hline 2 & $2 b$ & $\mathrm{Pr}$ & $\mathrm{Pr}$ & 1 & 65 \\
\hline 3 & 2c & $\mathrm{Pr}$ & $-\mathrm{CH}_{2} \mathrm{CH}_{2} \mathrm{Cl}$ & 8 & 58 \\
\hline 4 & $2 d$ & $\operatorname{Pr}$ & $\mathrm{Bn}$ & 2.5 & 55 \\
\hline 5 & $2 e$ & $\mathrm{Me}$ & $\mathrm{Bn}$ & 2.5 & 61 \\
\hline 6 & $2 f$ & $\mathrm{Pr}$ & $\mathrm{Ph}$ & 14 & 54 \\
\hline 7 & $2 g$ & $\mathrm{Me}$ & $\mathrm{Ph}$ & 14 & 52 \\
\hline 8 & $2 \mathrm{~h}$ & $\mathrm{Ph}$ & $\mathrm{Me}$ & 10 & 50 \\
\hline 9 & $2 i$ & $4-\mathrm{Me}-\mathrm{C}_{6} \mathrm{H}_{4}$ & $\mathrm{Me}$ & 10 & 56 \\
\hline 10 & $2 j$ & $4-\mathrm{Br}-\mathrm{C}_{6} \mathrm{H}_{4}$ & $\mathrm{Me}$ & 10 & 55 \\
\hline 11 & $2 k$ & $4-\mathrm{F}-\mathrm{C}_{6} \mathrm{H}_{4}$ & Me & 10 & 53 \\
\hline 12 & 21 & $4-\mathrm{NO}_{2}-\mathrm{C}_{6} \mathrm{H}_{4}$ & $\mathrm{Me}$ & 14 & 46 \\
\hline 13 & $2 m$ & $\mathrm{Me}$ & Me & 1 & 66 \\
\hline 14 & $2 n$ & $\mathrm{CH}_{2} \mathrm{Cl}$ & Me & 1 & 59 \\
\hline 15 & 20 & $-\mathrm{CHClCH}_{3}$ & Me & 2 & 4 \\
\hline 16 & $2 p$ & $\operatorname{Pr}$ & $2,4-\left(\mathrm{NO}_{2}\right)_{2}-\mathrm{C}_{6} \mathrm{H}_{3}$ & 14 & no reaction $d$ \\
\hline 17 & $2 q$ & $\mathrm{CF}_{3}$ & $\mathrm{Me}$ & 14 & no reaction $d$ \\
\hline 18 & $2 r$ & $\mathrm{Pr}$ & $t-\mathrm{Bu}$ & 2 & no target product \\
\hline
\end{tabular}

a All reactions were carried out on a $2.00 \mathrm{mmol}$ scale.

${ }^{b}$ The time for full conversion of 5-chloro- $1 \mathrm{H}$-pyrazole was monitored by TLC.

${ }^{c}$ All yields refer to isolated and purified products.

d The starting pyrazoles were isolated in quantitative yield.

e Conversion of the starting pyrazole was $100 \%$ and $1 \mathrm{H}$-pyrazole-4-carbaldehyde was not formed.

We concluded that the aromatic substituents, being more electron-withdrawing than the alkyl groups, prevent the formylation of 5-chloropyrazoles. This is especially evident for the nitrophenyl-substituted derivative $\mathbf{1 l}$ (Entry 12).

It should be noted that only a single example of formylation of 5 -chloropyrazole $\mathbf{1 g}$ with the $\mathrm{POCl}_{3} / \mathrm{DMF}$ system was reported. ${ }^{35}$ Our attempts to reproduce this experiment under the conditions described failed. In contrast, the application of optimal conditions found in our study allowed us to prepare the target formylated pyrazole in moderate yield (Entry 7 ).

We hypothesized that such yields of pyrazoles $2 \mathbf{f}, \mathbf{g}$ can be explained by formation of by-products. In fact, when the reaction of substrate $1 \mathrm{f}$ with $\mathrm{DMF}$ and $\mathrm{POCl}_{3}$ was conducted under optimal conditions, the target pyrazole $\mathbf{2 f}(52 \%)$ was isolated together with a minor amount of heterocycle $\mathbf{3}(6 \%)$. Its formation seems to be the result of the reaction of initial substrate 1 f with formaldehyde which was generated in situ in small quantity under long heating of DMF (Scheme 3). 


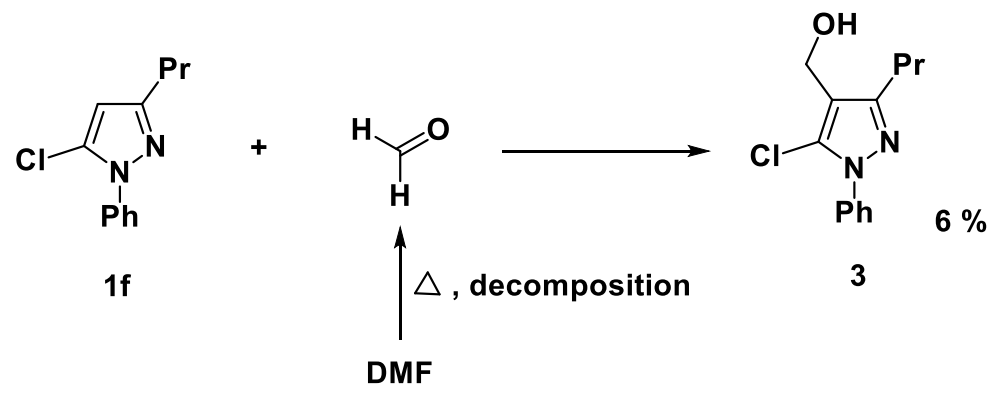

Scheme 3. Hydroxymethylation of 5-chloro-1-phenyl-3-propyl-1H-pyrazole 1 f.

Formylation of 5-chloro-1-(2-hydroxyethyl)-3-propylpyrazole $1 \mathrm{c}$ is accompanied by substitution of the hydroxyl group by chlorine atom to afford 5-chloro-1-(2-chloroethyl)-3-propyl-1H-pyrazole-4-carbaldehyde 2c in $58 \%$ yield (Entry 3 ).

An unexpected result was obtained when 5-chloro-3-(1-chloroethyl)-1-methyl-1H-pyrazole 10 was treated with DMF and $\mathrm{POCl}_{3}$ under optimal conditions. In this case the formylated pyrazole 20 is a minor product while the pyrazole 5 was isolated in $72 \%$ yield. We assumed that the latter compound was formed in one-pot tandem reaction sequence via elimination of the hydrogen chloride ${ }^{30}$ and following formylation of vinyl moiety of intermediate $\mathbf{A}$, as we have described in our previous work ${ }^{36}$. The target pyrazole 20 undergoes also dehydrochlorination under reaction conditions to afford 5-chloro-1-methyl-3-vinyl-1H-pyrazole-4carbaldehyde 4 in $7 \%$ yield (Scheme 4).
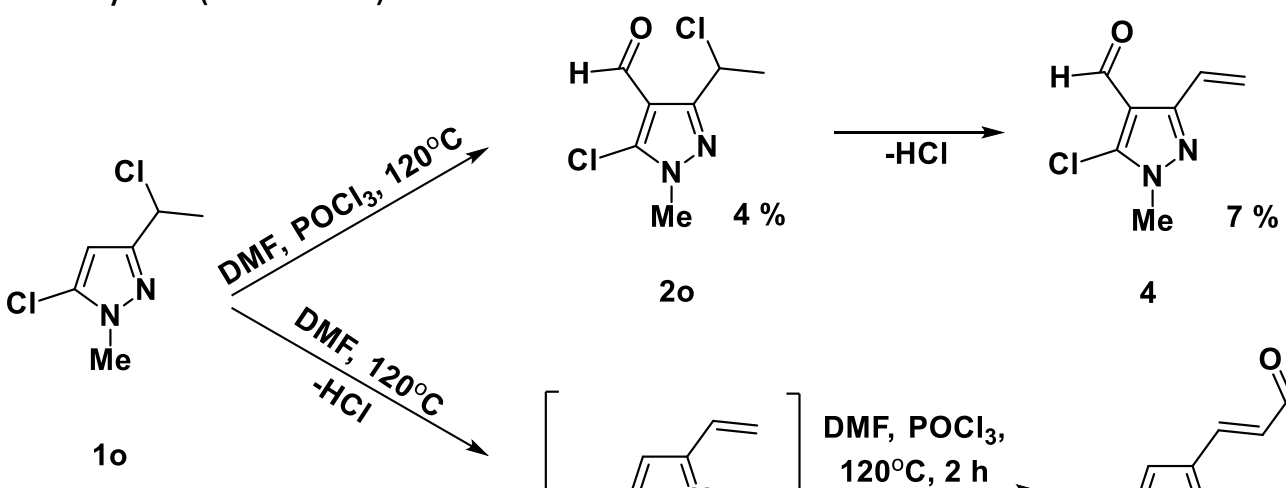

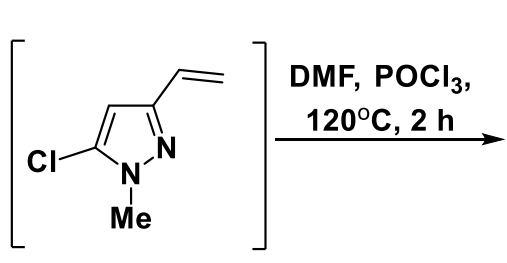

A

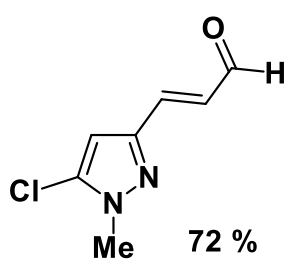

5

Scheme 4. Reactions of 5-chloro-3-(1-chloroethyl)-1-methyl-1H-pyrazole 10 under Vilsmeier-Haack conditions.

The reaction's scope is quite broad but pyrazoles 1p-r bearing strong electron-withdrawing groups on benzene ring or bulky moiety showed a low reactivity. Thus, the conversion of $\mathbf{1 p , q}$ achieved only $5 \%$ after refluxing of the mixture for a long time (entries 16,17). Finally, with pyrazole $1 \mathbf{r}$ the dealkylation reaction was observed: only a mixture of tautomers 6 was isolated (Scheme 5). This heterocycle does not afford formylated pyrazole under selected conditions. ${ }^{37}$ 


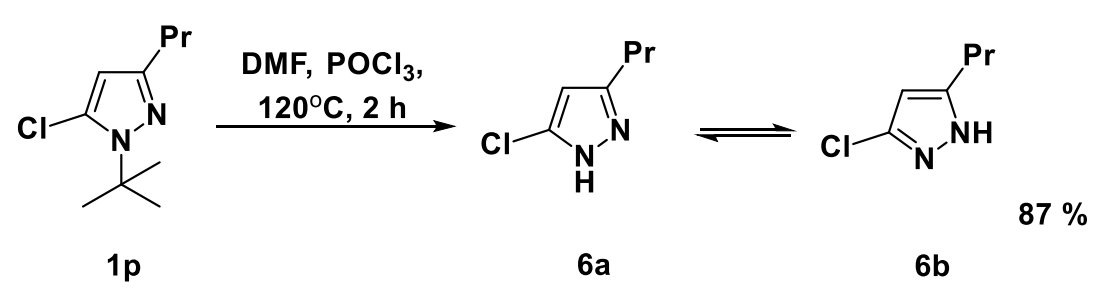

Scheme 5. Dealkylation of 1-(tert-butyl)-5-chloro-3-propyl-1H-pyrazole 1p.

\section{Conclusions}

An efficient method for the synthesis of previously unknown or hardly accessible 4-formylpyrazoles containing also a chlorine atom in position 5 has been developed. A number of different 5-chloro-4-formylpyrazoles bearing diverse substitution patterns can be synthesized by this method. The simplicity of execution, ready availability of starting materials and importance of the prepared pyrazoles make this procedure attractive for synthetic chemists. The method supplements the known approaches to halo-substituted formylpyrazoles and opens further routes to a variety of pyrazole derivatives, promising for the study of reactivity and possessing numerous valuable properties.

\section{Experimental Section}

General. The ${ }^{1} \mathrm{H},{ }^{13} \mathrm{C}$ and ${ }^{15} \mathrm{~N}$ NMR spectra were recorded in $\mathrm{CDCl}_{3}$ solutions at room temperature on Bruker DPX-400 and AV-400 spectrometers $\left(400.13,100.61\right.$ and $40.56 \mathrm{MHz}$, respectively). ${ }^{1} \mathrm{H},{ }^{13} \mathrm{C}$ and ${ }^{15} \mathrm{~N}$ Chemical shifts ( $\delta$ in ppm) were measured with accuracy of $0.01,0.02$ and $0.1 \mathrm{ppm}$, respectively, and referred to TMS $\left({ }^{1} \mathrm{H},{ }^{13} \mathrm{C}\right)$ and nitromethane $\left({ }^{15} \mathrm{~N}\right)$. The assignment of ${ }^{1} \mathrm{H}$ and ${ }^{13} \mathrm{C}$ signals in spectra was performed using $2 \mathrm{D}$ heteronuclear correlation HMBC-gp and HSQC-gp ${ }^{13} \mathrm{C}-{ }^{1} \mathrm{H}$ methods. The values of the $\delta{ }^{15} \mathrm{~N}$ were obtained through the $2 \mathrm{D}{ }^{1} \mathrm{H}-{ }^{15} \mathrm{~N} \mathrm{HMBC}$-gp experiment. Coupling constants $(\mathrm{J}$ in $\mathrm{Hz}$ ) values approaches to $0.1 \mathrm{~Hz}$.

IR spectra were recorded on a Bruker Vertex-70 instrument. MS analyses were recorded on a Shimadzu GCMSQP5050A instrument (ionization potential $70 \mathrm{eV}$ ). Column and thin-layer chromatography were carried out on commercial available $\mathrm{SiO}_{2}$ (Sigma-Aldrich).

Commercially available acyl chlorides, vinylidene chloride and hydrazines of the company Sigma-Aldrich were used. Alkyl hydrazines were distilled before reactions. Synthesis of 2,2-dichlorovinylketones and pyrazoles 1a, $\mathbf{g}-\mathbf{j}, \mathbf{I}-\mathbf{r}$ was presented in previous work. ${ }^{27-34}$ Pyrazoles $\mathbf{1 b - f , k}$ are described here for the first time.

3,3-Dichloro-1-(4-fluorophenyl)prop-2-en-1-one. A solution of a 4-fluorobenzoyl chloride $(4.758 \mathrm{~g}, 30 \mathrm{mmol})$ in $\mathrm{CH}_{2} \mathrm{Cl}_{2}(50 \mathrm{~mL})$ and $\mathrm{AlCl}_{3} 4(4.0 \mathrm{~g}, 30 \mathrm{mmol})$ was shaken for $20 \mathrm{~min}$ at $-5^{\circ} \mathrm{C}$. Then vinylidene chloride (3.490 $\mathrm{g}, 36 \mathrm{mmol}$ ) was added dropwise over a period of $20 \mathrm{~min}$ to avoid the temperature exceeding of $0^{\circ} \mathrm{C}$. After that, the reaction mass was stirred for $4 \mathrm{~h}$ at $\mathrm{rt}$, poured upon ice. An organic layer was separated and a water layer was extracted with $\mathrm{CH}_{2} \mathrm{Cl}_{2}(3 \times 50 \mathrm{~mL})$. The collected organic layer were dried over $\mathrm{MgSO}_{4}$ and evaporated under reduced pressure. The residual mass was dissolved in diethyl ether $(30 \mathrm{~mL})$, treated with triethylamine $(3.036 \mathrm{~g}, 30 \mathrm{mmol})$ at $-5^{\circ} \mathrm{C}$ for $10 \mathrm{~min}$ and kept to $\mathrm{rt}$, filtered and evaporated. The crude product was purified by silica gel column chromatography to give 3,3-dichloro-1-(4-fluorophenyl)prop-2-en-3-one, $4.995 \mathrm{~g}$ (76\%), $R_{f}=0.70$ (trichloromethane), colorless needles, m.p. $29^{\circ} \mathrm{C}$. [Lit. $\left.{ }^{38} 29-29.9^{\circ} \mathrm{C}\right]^{1} \mathrm{H} \mathrm{NMR}\left(400.13 \mathrm{MHz}, \mathrm{CDCl}_{3}\right): \delta 7.96-$ 
$7.93(\mathrm{~m}, 2 \mathrm{H}), 7.22(\mathrm{~s}, 1 \mathrm{H}), 7.17-7.13(\mathrm{~m}, 2 \mathrm{H}) \mathrm{ppm} .{ }^{13} \mathrm{C} \mathrm{NMR}\left(100.61 \mathrm{MHz}, \mathrm{CDCl}_{3}\right): \delta 185.0,166.0\left(\mathrm{~d},{ }^{1} J_{\mathrm{C}, \mathrm{F}} 256.0\right.$ $\mathrm{Hz}), 135.6,133.2\left(\mathrm{~d},{ }^{4} J_{C, F} 2.4 \mathrm{~Hz}\right), 131.1\left(\mathrm{~d},{ }^{3} J_{C, F} 9.6 \mathrm{~Hz}\right), 123.8,116.0\left(\mathrm{~d},{ }^{2} J_{C, F} 22.0 \mathrm{~Hz}\right) \mathrm{ppm}$. Anal. calcd. for $\mathrm{C}_{9} \mathrm{H}_{5} \mathrm{Cl}_{2} \mathrm{FO}: \mathrm{C}, 49.35 ; \mathrm{H}, 2.30 ; \mathrm{Cl}, 32.37$. Found \%: $\mathrm{C}, 49.24 ; \mathrm{H}, 2.32 ; \mathrm{Cl}, 32.45$.

General procedure for the synthesis of $\mathbf{1 H}$-pyrazoles (1). A triethylamine $(2 \mathrm{mmol})$ was added to a solution of dichlorovinylketone $(2 \mathrm{mmol})$ in diethyl ether $(10 \mathrm{~mL})$ under cooling with ice-water bath. After that a hydrazine $(2 \mathrm{mmol}$ ) was added dropwise for $20 \mathrm{~min}$. In the synthesis of $N$-methylpyrazole $1 \mathrm{k} 2$ equiv (4 mmol) of dimethylhydrazine was used without using of triethylamine. The reaction mixture was stirred for $1.5 \mathrm{~h}$ at room temperature. The reaction mixture was filtered off and diethyl ether was evaporated. Individual pyrazoles usable for further purposes without additional purification were obtained.

5-Chloro-1,3-dipropyl-1H-pyrazole (1b). The general procedure was followed using triethylamine $(2 \mathrm{mmol})$, 1,1-dichlorohex-1-en-3-one $(2 \mathrm{mmol})$ and propylhydrazine $(2 \mathrm{mmol})$, reaction time: $1.5 \mathrm{~h}, 344 \mathrm{mg}(92 \%)$, yellow oil. IR (film): 3186, 3123, 2963, 2875, 1724, 1516, 1461, $773 \mathrm{~cm}^{-1} .{ }^{1} \mathrm{H} \mathrm{NMR}\left(400.13 \mathrm{MHz}, \mathrm{CDCl}_{3}\right): \delta 5.95$ $(\mathrm{s}, 1 \mathrm{H}), 4.01(\mathrm{t}, 2 \mathrm{H}, J 7.2 \mathrm{~Hz}), 2.52(\mathrm{t}, 2 \mathrm{H}, J 7.6 \mathrm{~Hz}), 1.81(\mathrm{~m}, 2 \mathrm{H}), 1.61(\mathrm{~m}, 2 \mathrm{H}), 0.93(\mathrm{t}, 3 \mathrm{H}, J 7.3 \mathrm{~Hz}), 0.89(\mathrm{t}, 3 \mathrm{H}$, J 7.4 Hz) ppm. ${ }^{13} \mathrm{C} \mathrm{NMR}\left(100.61 \mathrm{MHz}, \mathrm{CDCl}_{3}\right): \delta 152.8,126.4,102.8,50.2,30.6,23.2,22.7,13.7,10.9$ ppm. Anal. calcd. for $\mathrm{C}_{9} \mathrm{H}_{15} \mathrm{ClN}_{2}$ : C, 57.91; H, 8.10; $\mathrm{Cl}, 18.99 ; \mathrm{N}, 15.01$. Found \%: $\mathrm{C}, 57.81 ; \mathrm{H}, 8.06 ; \mathrm{Cl}, 19.06 ; \mathrm{N}, 15.07$.

2-(5-chloro-3-propyl-1H-pyrazol-1-yl)ethan-1-ol (1c). The general procedure was followed using triethyla mine (2 mmol), 1,1-dichlorohex-1-en-3-one $(2 \mathrm{mmol})$ and 2-hydrazineylethan-1-ol $(2 \mathrm{mmol})$, reaction time: $1.5 \mathrm{~h}$, $328 \mathrm{mg}$ (87\%) orange oil. IR (film): 3326, 3131, 2958, 2873, 1516, 1463, $775 \mathrm{~cm}^{-1} .{ }^{1} \mathrm{H} \mathrm{NMR}\left(400.13 \mathrm{MHz}, \mathrm{CDCl}_{3}\right)$ : $\delta 6.03(\mathrm{~s}, 1 \mathrm{H}), 4.17(\mathrm{t}, 2 \mathrm{H}), 3.98(\mathrm{t}, 2 \mathrm{H}), 2.54(\mathrm{t}, 2 \mathrm{H}, J 7.5 \mathrm{~Hz}), 1.64(\mathrm{~m}, 2 \mathrm{H}), 0.95(\mathrm{t}, 3 \mathrm{H}, J 7.4 \mathrm{~Hz}) \mathrm{ppm} .{ }^{13} \mathrm{C} \mathrm{NMR}$ $\left(100.61 \mathrm{MHz}, \mathrm{CDCl}_{3}\right): \delta$ 153.6, 127.3, 103.2, 61.4, 49.9, 30.6, 22.5, 13.8 ppm. Anal. calcd. for $\mathrm{C}_{8} \mathrm{H}_{13} \mathrm{ClN}_{2} \mathrm{O}: \mathrm{C}$, $50.93 ; \mathrm{H}, 6.95 ; \mathrm{Cl}, 18.79 ; \mathrm{N}, 14.85$. Found \%: C, 51.01; $\mathrm{H}, 6.93 ; \mathrm{Cl}, 18.70 ; \mathrm{N}, 14.90$.

1-Benzyl-5-chloro-3-propyl-1H-pyrazole (1d). The general procedure was followed using triethylamine $(2$ mmol), 1,1-dichlorohex-1-en-3-one $(2 \mathrm{mmol})$ and benzylhydrazine $(2 \mathrm{mmol})$, reaction time: $1.5 \mathrm{~h}, 418 \mathrm{mg}$ (89\%), orange oil. IR (film): 3127, 3063, 2959, 2873, 1708, 1574, 1457, $724 \mathrm{~cm}^{-1} .{ }^{1} \mathrm{H} \mathrm{NMR}\left(400.13 \mathrm{MHz}, \mathrm{CDCl}_{3}\right)$ : $\delta$ 7.25-7.39 (m, 5H), $6.12(\mathrm{~s}, 1 \mathrm{H}), 5.36(\mathrm{~s}, 2 \mathrm{H}), 1.64(\mathrm{t}, 2 \mathrm{H}, J 7.6 \mathrm{~Hz}), 1.72(\mathrm{~m}, 2 \mathrm{H}), 1.02(\mathrm{t}, 3 \mathrm{H}, J 7.3 \mathrm{~Hz}) \mathrm{ppm} .{ }^{13} \mathrm{C}$ NMR $\left(100.61 \mathrm{MHz} \mathrm{CDCl}_{3}\right): \delta$ 153.3, 136.2, 128.6, 127.7, 127.1, 126.3, 103.6, 52.3, 30.5, 22.6, 13.7 ppm. Anal. calcd. for $\mathrm{C}_{13} \mathrm{H}_{15} \mathrm{ClN}_{2}: \mathrm{C}, 66.52 ; \mathrm{H}, 6.44 ; \mathrm{Cl}, 15.10 ; \mathrm{N}, 11.93$. Found \%: $\mathrm{C}, 66.62 ; \mathrm{H}, 6.42 ; \mathrm{Cl}, 15.06 ; \mathrm{N}, 11.90$.

1-Benzyl-5-chloro-3-methyl-1H-pyrazole (1e). The general procedure was followed using triethylamine $(2$ mmol), 4,4-dichlorobut-3-en-2-one $(2 \mathrm{mmol})$ and benzylhydrazine $(2 \mathrm{mmol})$, reaction time: $1.5 \mathrm{~h}, 343 \mathrm{mg}$ (83\%), orange oil. IR (film): 3128, 3033, 2932, 1605, 1518, 1451, $776 \mathrm{~cm}^{-1} .{ }^{1} \mathrm{H} \mathrm{NMR}\left(400.13 \mathrm{MHz}, \mathrm{CDCl}_{3}\right): \delta 7.25-$ $7.38(\mathrm{~m}, 5 \mathrm{H}), 6.08(\mathrm{~s}, 1 \mathrm{H}), 5.32(\mathrm{~s}, 2 \mathrm{H}), 2.30(\mathrm{~s}, 3 \mathrm{H}) \mathrm{ppm} .{ }^{13} \mathrm{C} \mathrm{NMR}\left(100.61 \mathrm{MHz}, \mathrm{CDCl}_{3}\right): \delta 148.6,136.2,128.5$, 127.7, 127.1, 127.0, 104.4, 52.3, 13.9 ppm. Anal. calcd. for $\mathrm{C}_{11} \mathrm{H}_{11} \mathrm{CIN}_{2}$ : C, 63.93; $\mathrm{H}, 5.36 ; \mathrm{Cl}, 17.15 ; \mathrm{N}, 13.55$. Found \%: C, 63.80; $\mathrm{H}, 5.34 ; \mathrm{Cl}, 17.22 ; \mathrm{N}, 13.64$.

5-chloro-1-phenyl-3-propyl-1H-pyrazole (1f). The general procedure was followed using triethylamine $(2$ $\mathrm{mmol})$, 1,1-dichlorohex-1-en-3-one $(2 \mathrm{mmol})$ and phenylhydrazine $(2 \mathrm{mmol})$, reaction time: $1.5 \mathrm{~h}, 353 \mathrm{mg}$ (80\%), yellow oil. IR (film): 3121, 2058, 2959, 2870, 1597, 1523, 1453, $763 \mathrm{~cm}^{-1} .{ }^{1} \mathrm{H} \mathrm{NMR}(400.13 \mathrm{MHz}, \mathrm{CDCl}): \delta$ 7.58-7.35 (m, 5H), $6.21(\mathrm{~s}, 1 \mathrm{H}), 2.64(\mathrm{t}, 2 \mathrm{H}, J 7.6 \mathrm{~Hz}), 1.72(\mathrm{~m}, 2 \mathrm{H}), 1.01(\mathrm{t}, 3 \mathrm{H}, J 7.3 \mathrm{~Hz}) \mathrm{ppm} .{ }^{13} \mathrm{C}$ NMR $(100.61$ $\left.\mathrm{MHz}, \mathrm{CDCl}_{3}\right): \delta 154.3,138.3,128.8,127.8,126.7,124.8,105.3,30.6,22.5,13.8$ ppm. Anal. calcd. for $\mathrm{C}_{12} \mathrm{H}_{13} \mathrm{ClN}_{2}$ : C, 65.31; H, 5.94; Cl, 16.06; N, 12.69. Found \%: C, 65.40; H, 5.96; Cl, 16.00; N, 12.64 .

5-Chloro-3-(4-fluorophenyl)-1-methyl-1H-pyrazole (1k). The general procedure was followed using 3,3dichloro-1-(4-fluorophenyl)prop-2-en-1-one $(2 \mathrm{mmol})$ and 1,1-dimethylhydrazine (4 mmol), reaction time: 1.5 h, $345 \mathrm{mg}$ (82\%), beige solid, m.p. 50-52 ${ }^{\circ} \mathrm{C}$. IR (film): $\cup$ 3125, 3058, 2944, 2877, 1658, 1603, $1497,779 \mathrm{~cm}^{-1} .{ }^{1} \mathrm{H}$ NMR $\left(400.13 \mathrm{MHz} \mathrm{CDCl}_{3}\right): \delta 7.72-7.69(\mathrm{~m}, 2 \mathrm{H}), 7.11-7.07(\mathrm{~m}, 2 \mathrm{H}), 6.45(\mathrm{~s}, 1 \mathrm{H}), 3.89(\mathrm{~s}, 3 \mathrm{H}) \mathrm{ppm} .{ }^{13} \mathrm{C} \mathrm{NMR}$ 
(100.61 MHz, CDCl 3 ): $\delta 162.7$ (d, ${ }^{1} J_{C, F} 247.3 \mathrm{~Hz}$ ), 149.9, 129.0 (d, $\left.{ }^{4} J_{C, F} 2.8 \mathrm{~Hz}\right), 128.2,127.0\left(\mathrm{~d},{ }^{3} J_{C, F} 8.4 \mathrm{~Hz}\right), 115.5$ $\left(d,{ }^{2} J_{C, F} 21.6 \mathrm{~Hz}\right), 101.5,36.2$ ppm. Anal. calcd. for $\mathrm{C}_{10} \mathrm{H}_{8} \mathrm{ClFN}_{2}: \mathrm{C}, 57.02 ; \mathrm{H}, 3.83 ; \mathrm{Cl}, 16.83 ; \mathrm{N}, 13.30$. Found \%: $\mathrm{C}$, $56.85 ; \mathrm{H}, 3.84 ; \mathrm{Cl}, 16.87 ; \mathrm{N}, 13.34$.

General procedure for the synthesis of $\mathbf{1 H}$-pyrazole-4-carbaldehydes (2). $\mathrm{POCl}_{3}$ (4 equiv.) was added to DMF ( 6 equiv.) at $0{ }^{\circ} \mathrm{C}$. After 10-15 min, pyrazole (1.0 equiv.) was added to the reaction mixture, which was then stirred at $120^{\circ} \mathrm{C}$ until the pyrazole was completely consumed (based on TLC analysis). The reaction was quenched with water and was neutralized with a saturated solution of $\mathrm{Na}_{2} \mathrm{CO}_{3}$ to $\mathrm{pH}^{\sim}$. The mixture was extracted with chloroform ( 3 times). The combined organic layers were dried with $\mathrm{Mg}_{2} \mathrm{SO}_{4}$, and filtered. The solvent was removed under vacuum and the residue was purified by silica gel column chromatography (diethyl ether/hexane) to afford the $1 H$-pyrazole-4-carbaldehyde.

5-Chloro-1-methyl-3-propyl-1H-pyrazole-4-carbaldehyde (2a). The general procedure was followed using 5chloro-1,3-dimethyl-1H-pyrazole $1 \mathrm{a}(2 \mathrm{mmol})$, reaction time: $1 \mathrm{~h}, 250 \mathrm{mg}(67 \%)$ orange oil, $R_{f} 0.33$ (diethyl ether/hexane, $1: 1 \mathrm{v} / \mathrm{v})$. IR (film): 2961, 2931, 2873, 1682, 1510, 1470, $772 \mathrm{~cm}^{-1} .{ }^{1} \mathrm{H} \mathrm{NMR}(400.13 \mathrm{MHz}, \mathrm{CDCl} 3): \delta$ $9.85(\mathrm{~s}, 1 \mathrm{H}), 3.83(\mathrm{~s}, 3 \mathrm{H}), 2.81(\mathrm{t}, 2 \mathrm{H}, J 7.3 \mathrm{~Hz}), 1.67(\mathrm{~m}, 2 \mathrm{H}), 0.97(\mathrm{t}, 3 \mathrm{H}, J 7.0 \mathrm{~Hz}) \mathrm{ppm} .{ }^{13} \mathrm{C} \mathrm{NMR}(100.61 \mathrm{MHz}$, $\left.\mathrm{CDCl}_{3}\right): \delta 183.2,154.9,133.6,115.8,35.9,29.8,21.8,13.8$ ppm. ${ }^{15} \mathrm{~N} \mathrm{NMR}\left(40.56 \mathrm{MHz} \mathrm{CDCl}_{3}\right): \delta-75.8,-180.7$ ppm. MS (El, 70 eV): m/z (\%) 186 (98, [M+]), 171 (85), 157 (81), 130 (100), 76 (45). Anal. calcd. for $\mathrm{C}_{8} \mathrm{H}_{11} \mathrm{ClN}_{2} \mathrm{O}$ : C, 51.48; H, 5.94; Cl, 19.00; N, 15.01. Found \%: C, 51.62; H, 5.95; Cl, 18.95; N, 14.98.

5-Chloro-1,3-dipropyl-1H-pyrazole-4-carbaldehyde (2b). The general procedure was followed using 5-chloro1,3-dipropyl-1H-pyrazole (1b, $2 \mathrm{mmol}$ ), reaction time: $1 \mathrm{~h}, 279 \mathrm{mg}$ (65\%), orange oil, $R_{f} 0.30$ (diethyl ether/hexane, $1: 1 \mathrm{v} / \mathrm{v})$. IR (film): 2964, 2934, 2875, 1683, 1521, 1469, $776 \mathrm{~cm}^{-1} .{ }^{1} \mathrm{H} \mathrm{NMR}(400.13 \mathrm{MHz}, \mathrm{CDCl} 3): \delta$ $9.86(\mathrm{~s}, 1 \mathrm{H}), 4.08(\mathrm{t}, 2 \mathrm{H}, J 7.2 \mathrm{~Hz}), 2.82(\mathrm{t}, 2 \mathrm{H}, J 7.7 \mathrm{~Hz}), 1.83-1.93(\mathrm{~m}, 2 \mathrm{H}), 1.64-1.73(\mathrm{~m}, 2 \mathrm{H}), 0.92-0.98(\mathrm{~m}, 6 \mathrm{H})$ ppm. ${ }^{13} \mathrm{C}$ NMR $\left(100.61 \mathrm{MHz} \mathrm{CDCl}_{3}\right): \delta 183.3,154.9,133.1,115.6,50.5,29.9,22.8,21.9,13.8,10.9 \mathrm{ppm} .{ }^{15} \mathrm{~N}$ NMR (40.56 MHz, CDCl $): \delta$-77.4, -169.2 ppm. MS (El, $70 \mathrm{eV}): \mathrm{m} / \mathrm{z}(\%) 214$ (76, [M+]), 199 (50), $186(45), 179$ (60), 158 (100), 143 (55). Anal. calcd. for $\mathrm{C}_{10} \mathrm{H}_{15} \mathrm{CIN} 2 \mathrm{O}: \mathrm{C}, 55.94 ; \mathrm{H}, 7.04 ; \mathrm{Cl}, 16.51 ; \mathrm{N}, 13.05$. Found \%: $\mathrm{C}, 51.82$; $\mathrm{H}, 7.06 ; \mathrm{Cl}, 16.56 ; \mathrm{N}, 13.03$.

5-Chloro-1-(2-chloroethyl)-3-propyl-1H-pyrazole-4-carbaldehyde (2c). The general procedure was followed using 2-(5-chloro-3-propyl-1H-pyrazol-1-yl)ethanol (1c, $2 \mathrm{mmol})$, reaction time: $8 \mathrm{~h}, 273 \mathrm{mg}$ (58\%), colorless needles, m.p. $77-79{ }^{\circ} \mathrm{C}, R_{f} 0.16$ (diethyl ether/hexane, 1:2 v/v). IR (film): 2962, 2933, 2872, 1682, 1522, 1464, $778 \mathrm{~cm}^{-1} .{ }^{1} \mathrm{H}$ NMR $\left(400.13 \mathrm{MHz}^{\mathrm{CDCl}} 3\right): \delta 9.88(\mathrm{~s}, 1 \mathrm{H}), 4.43(\mathrm{t}, 2 \mathrm{H}, J 6.4 \mathrm{~Hz}), 3.91(\mathrm{t}, 2 \mathrm{H}, J 6.1 \mathrm{~Hz}), 2.83(\mathrm{t}, 2 \mathrm{H}, J$ $7.8 \mathrm{~Hz}), 1.64-1.73(\mathrm{~m}, 2 \mathrm{H}), 0.96(\mathrm{t}, 3 \mathrm{H}, J 7.3 \mathrm{~Hz}) \mathrm{ppm} .{ }^{13} \mathrm{C} N M R\left(100.61 \mathrm{MHz}, \mathrm{CDCl}_{3}\right): \delta 183.2,155.7,134.4$, 115.9, 49.7, 41.4, 29.9, 21.6, 13.7 ppm. ${ }^{15} \mathrm{~N} \mathrm{NMR} \mathrm{(40.56} \mathrm{MHz,} \mathrm{CDCl} 3$ ) $\delta-78.5,-176.8 \mathrm{ppm} . \mathrm{MS}$ (El, $\left.70 \mathrm{eV}\right): \mathrm{m} / \mathrm{z}$ (\%) 234 (60, [M+]), 219 (62), 206 (60), 178 (100), 157 (56), 143 (52), 63 (88). Anal. calcd. for $\mathrm{C}_{9} \mathrm{H}_{12} \mathrm{Cl}_{2} \mathrm{~N}_{2} \mathrm{O}: \mathrm{C}_{\text {, }}$ 45.98; $\mathrm{H}, 5.14 ; \mathrm{Cl}, 30.16 ; \mathrm{N}, 11.91$. Found \%: C, 46.10; $\mathrm{H}, 5.14 ; \mathrm{Cl}, 30.08 ; \mathrm{N}, 11.90$.

1-Benzyl-5-chloro-3-propyl-1H-pyrazole-4-carbaldehyde (2d). The general procedure was followed using 1benzyl-5-chloro-3-propyl-1H-pyrazole (1d, $2 \mathrm{mmol}$ ), reaction time: $2.5 \mathrm{~h}, 289 \mathrm{mg}$ (55\%), orange powder, mp 35 ${ }^{\circ} \mathrm{C}, R_{f} 0.47$ (diethyl ether/hexane, 1:1 v/v). IR (film): u 3066, 2962, 2932, 2873, 1683, 1521, 1463, $775 \mathrm{~cm}^{-1} .{ }^{1} \mathrm{H}$ NMR (400.13 MHz, $\left.\mathrm{CDCl}_{3}\right): \delta 9.88(\mathrm{~s}, 1 \mathrm{H}), 7.25-7.37(\mathrm{~m}, 5 \mathrm{H}), 5.33(\mathrm{~s}, 2 \mathrm{H}), 2.85(\mathrm{t}, 2 \mathrm{H}, J \mathrm{~J} 7.7 \mathrm{~Hz}), 1.67-1.76(\mathrm{~m}$, $2 \mathrm{H}), 0.98(\mathrm{t}, 3 \mathrm{H}, J 7.4 \mathrm{~Hz}) \mathrm{ppm} .{ }^{13} \mathrm{C}$ NMR $\left(100.61 \mathrm{MHz}, \mathrm{CDCl}_{3}\right): \delta 183.2,155.2,134.8,133.4,128.9,128.3,127.5$, 116.1, 52.7, 29.8, 21.8, 13.8 ppm. ${ }^{15} \mathrm{~N}$ NMR (40.56 MHz, CDCl 3$): \delta-75.6,-170.4$ ppm. MS (El, $\left.70 \mathrm{eV}\right): \mathrm{m} / \mathrm{z}(\%)$ 262 (23, [M+]), 91 (100), 65 (34), 130 (100), 76 (45). Anal. calcd. for $\mathrm{C}_{14} \mathrm{H}_{15} \mathrm{ClN}_{2} \mathrm{O}: \mathrm{C}, 64.00 ; \mathrm{H}, 5.75 ; \mathrm{Cl}, 13.49 ; \mathrm{N}$, 10.66. Found \%: C, 64.18; $\mathrm{H}, 5.77 ; \mathrm{Cl}, 13.53 ; \mathrm{N}, 10.62$.

1-Benzyl-5-chloro-3-methyl-1H-pyrazole-4-carbaldehyde (2e). The general procedure was followed using 1benzyl-5-chloro-3-methyl-1H-pyrazole (1e, $2 \mathrm{mmol}$ ), reaction time: 2.5 h, $286 \mathrm{mg}$ (61\%), orange powder, m.p. 
101-102 ${ }^{\circ} \mathrm{C}, R_{f} 0.30$ (diethyl ether/hexane, 1:2 v/v). IR (film): 3062, 2961, 2849, 1681, 1526, 1460, $765 \mathrm{~cm}^{-1} .{ }^{1} \mathrm{H}$ NMR $\left(400.13 \mathrm{MHz}, \mathrm{CDCl}_{3}\right): \delta 9.87(\mathrm{~s}, 1 \mathrm{H}), 7.25-7.37(\mathrm{~m}, 5 \mathrm{H}), 5.30(\mathrm{~s}, 2 \mathrm{H}), 2.47(\mathrm{~s}, 3 \mathrm{H}) \mathrm{ppm} .{ }^{13} \mathrm{C} \mathrm{NMR}(100.61$ $\mathrm{MHz}_{\mathrm{CDCl}}$ ): $\delta$ 183.2, 151.0, 134.8, 133.5, 128.7, 128.2, 127.4, 116.3, 52.5 ppm. ${ }^{15} \mathrm{~N} \mathrm{NMR}\left(40.56 \mathrm{MHz}^{\mathrm{CDCl}}\right)_{3}: \delta$ -75.5, -170.4 ppm. MS (El, $70 \mathrm{eV}): m / z$ (\%) 234 (15, [M+]), 199 (11), 91 (100), 65 (17). Anal. calcd. for $\mathrm{C}_{12} \mathrm{H}_{11} \mathrm{ClN}_{2} \mathrm{O}: \mathrm{C}, 61.42 ; \mathrm{H}, 4.72 ; \mathrm{Cl}, 15.11 ; \mathrm{N}, 11.94$. Found \%: $\mathrm{C}, 61.35 ; \mathrm{H}, 4.75 ; \mathrm{Cl}, 15.07 ; \mathrm{N}, 11.98$.

5-Chloro-1-phenyl-3-propyl-1H-pyrazole-4-carbaldehyde (2f). The general procedure was followed using 5chloro-1-phenyl-3-propyl-1H-pyrazole (1f, 2 mmol), reaction time: 14 h, $269 \mathrm{mg}$ (54\%), light brown needles, m.p. 151-152 ${ }^{\circ} \mathrm{C}, R_{f} 0.23$ (diethyl ether/hexane, 1:8 v/v). IR (film): u 3064, 2951, 2923, 2854, 1675, 1525, 1462, $766 \mathrm{~cm}^{-1}$. ${ }^{1} \mathrm{H}$ NMR $\left(400.13 \mathrm{MHz}, \mathrm{CDCl}_{3}\right): \delta 9.97(\mathrm{~s}, 1 \mathrm{H}), 7.45-7.56(\mathrm{~m}, 5 \mathrm{H}), 2.90(\mathrm{t}, 2 \mathrm{H}, J 7.6 \mathrm{~Hz}), 1.70-1.79(\mathrm{~m}$, 2H), 1.00 (t, 3H, J $7.3 \mathrm{~Hz}$ ) ppm. ${ }^{13} \mathrm{C}$ NMR (100.61 MHz, $\left.\mathrm{CDCl}_{3}\right): \delta 183.6,155.7,136.9,133.4,129.2,129.0,125.1$, 116.9, 29.8, 21.6, 13.8 ppm. ${ }^{15} \mathrm{~N} \mathrm{NMR}\left(40.56 \mathrm{MHz}, \mathrm{CDCl}_{3}\right): \delta-73.0,-165.3 \mathrm{ppm} . \mathrm{MS}(\mathrm{El}, 70 \mathrm{eV}): \mathrm{m} / \mathrm{z}$ (\%) 248 (45, $[\mathrm{M}+]), 220$ (41), 192 (82), 133 (35), 77 (100). Anal. calcd. for $\mathrm{C}_{13} \mathrm{H}_{13} \mathrm{CIN} 2 \mathrm{O}: \mathrm{C}, 62.78 ; \mathrm{H}, 5.27 ; \mathrm{Cl}, 14.25 ; \mathrm{N}, 11.26$. Found \%: C, 62.60; $\mathrm{H}, 5.25 ; \mathrm{Cl}, 14.20 ; \mathrm{N}, 13.30$.

5-Chloro-3-methyl-1-phenyl-1H-pyrazole-4-carbaldehyde $(\mathbf{2 g})$. The general procedure was followed using 5chloro-3-methyl-1-phenyl-1H-pyrazole (1g, $2 \mathrm{mmol}$ ), reaction time: $14 \mathrm{~h}, 230 \mathrm{mg}$ (52\%), dark red needle, m.p. 142-143 ${ }^{\circ} \mathrm{C}, R_{f} 0.17$ (diethyl ether/hexane, 1:5 v/v). IR (film): 3065, 2927, 1676, 1527, 1469, $764 \mathrm{~cm}^{-1} .{ }^{1} \mathrm{H} \mathrm{NMR}$ (400.13 MHz, $\left.\mathrm{CDCl}_{3}\right): \delta 9.97(\mathrm{~s}, 1 \mathrm{H}), 7.46-7.55(\mathrm{~m}, 5 \mathrm{H}), 2.54(\mathrm{~s}, 3 \mathrm{H})$ ppm. ${ }^{13} \mathrm{C} \mathrm{NMR}\left(100.61 \mathrm{MHz}, \mathrm{CDCl}_{3}\right): \delta 183.8$, 151.7, 136.8, 133.4, 129.2, 129.1, 125.1, 117.3, 13.8 ppm. ${ }^{15} \mathrm{~N} \mathrm{NMR}\left(40.56 \mathrm{MHz}, \mathrm{CDCl}_{3}\right): \delta-72.9,-165.1 \mathrm{ppm}$. MS (El, $70 \mathrm{eV}): m / z(\%) 220$ (93, [M+]), 219 (100), 155 (22), 77 (63), 51 (50). Anal. calcd. for $\mathrm{C}_{11} \mathrm{H}_{9} \mathrm{CIN} \mathrm{N}_{2} \mathrm{O}: \mathrm{C}$, 59.88; $\mathrm{H}, 4.11 ; \mathrm{Cl}, 16.07 ; \mathrm{N}, 12.70$. Found \%: C, 59.73; $\mathrm{H}, 4.09 ; \mathrm{Cl}, 16.10 ; \mathrm{N}, 12.73$.

5-Chloro-1-methyl-3-phenyl-1H-pyrazole-4-carbaldehyde (2h). The general procedure was followed using 5chloro-1-methyl-3-phenyl-1H-pyrazole (1h, $2 \mathrm{mmol}$ ), reaction time: $14 \mathrm{~h}, 221 \mathrm{mg}$, (50\%) beige oil, $R_{f} 0.50$ (diethyl ether/hexane, 1:1 v/v). IR (film): 3062, 2946, 1683, 1502, 1448, $779 \mathrm{~cm}^{-1} .{ }^{1} \mathrm{H} \mathrm{NMR}(400.13 \mathrm{MHz}$, $\left.\mathrm{CDCl}_{3}\right): \delta 9.92(\mathrm{~s}, 1 \mathrm{H}), 7.72-7.73(\mathrm{~m}, 2 \mathrm{H}), 7.43-7.45(\mathrm{~m}, 3 \mathrm{H}), 3.90(\mathrm{~s}, 3 \mathrm{H}) \mathrm{ppm} .{ }^{13} \mathrm{C} \mathrm{NMR}\left(100.61 \mathrm{MHz}, \mathrm{CDCl}_{3}\right): \delta$ 183.3, 153.3, 133.1, 130.9, 129.2, 128.6, 128.4, 115.3, 36.2 ppm. ${ }^{15} \mathrm{~N} \mathrm{NMR}\left(40.56 \mathrm{MHz}, \mathrm{CDCl}_{3}\right) \delta-75.0,-176.7$ ppm. MS (El, $70 \mathrm{eV}): \mathrm{m} / \mathrm{z}(\%) 220(100,[\mathrm{M}+])$. Anal. calcd. for $\mathrm{C}_{11} \mathrm{H}_{9} \mathrm{ClN} \mathrm{N}_{2} \mathrm{O}: \mathrm{C}, 59.88 ; \mathrm{H}, 4.11 ; \mathrm{Cl}, 16.07 ; \mathrm{N}, 12.70$. Found \%: C, 59.71; $\mathrm{H}, 4.10 ; \mathrm{Cl}, 16.08 ; \mathrm{N}, 12.75$.

5-Chloro-1-methyl-3-(4-methylphenyl)-1H-pyrazole-4-carbaldehyde (2i). The general procedure was followed using 5-chloro-1-methyl-3-(4-methylphenyl)-1H-pyrazole (1i, $2 \mathrm{mmol}$ ), reaction time: $14 \mathrm{~h}, 263 \mathrm{mg}$ (56\%), beige powder, $\mathrm{mp} 105-10{ }^{\circ} \mathrm{C}, R_{f} 0.33$ (diethyl ether/hexane, 1:1 v/v). IR (film): 3058, 2920, 1673, 1492, 1451, $830 \mathrm{~cm}^{-1} .{ }^{1} \mathrm{H}$ NMR $\left(400.13 \mathrm{MHz}, \mathrm{CDCl}_{3}\right) \delta 9.95(\mathrm{~s}, 1 \mathrm{H}), 7.62-7.64(\mathrm{~m}, 2 \mathrm{H}), 7.27-7.29(\mathrm{~m}, 2 \mathrm{H}), 3.94(\mathrm{~s}, 3 \mathrm{H}), 2.42(\mathrm{~s}$, $3 \mathrm{H}) \mathrm{ppm} .{ }^{13} \mathrm{C} \mathrm{NMR}\left(100.61 \mathrm{MHz}, \mathrm{CDCl}_{3}\right): \delta 183.6,153.6,139.4,133.0,129.2,128.6,128.4,115.3,36.2 \mathrm{ppm} .{ }^{15} \mathrm{~N}$ NMR (40.56 MHz, CDCl $): \delta$-75.8, -177.8 ppm. MS (El, $70 \mathrm{eV}): \mathrm{m} / \mathrm{z}(\%) 234$ (100, [M+]), 219 (66), $116(28), 91$ (23), 76 (19). Anal. calcd. for $\mathrm{C}_{12} \mathrm{H}_{11} \mathrm{ClN}_{2} \mathrm{O}: \mathrm{C}, 61.41 ; \mathrm{H}, 4.72 ; \mathrm{Cl}, 15.11 ; \mathrm{N}, 11.94$. Found \%: $\mathrm{C}, 61.52 ; \mathrm{H}, 4.71 ; \mathrm{Cl}$, 15.08; N, 11.93.

3-(4-Bromophenyl)-5-chloro-1-methyl-1H-pyrazole-4-carbaldehyde (2j). The general procedure was followed using 3-(4-bromophenyl)-5-chloro-1-methyl-1H-pyrazole (1g, $2 \mathrm{mmol}$ ), reaction time: $10 \mathrm{~h}, 330 \mathrm{mg}$ (55\%), light yellow needles, $\mathrm{mp} 74-76{ }^{\circ} \mathrm{C}, R_{f} 0.23$ (diethyl ether/hexane, 1:2 v/v). IR (film): 3076, 2945, 1684, 1499, 1446, 834, $778 \mathrm{~cm}^{-1} .{ }^{1} \mathrm{H}$ NMR $\left(400.13 \mathrm{MHz}, \mathrm{CDCl}_{3}\right): \delta 9.94(\mathrm{~s}, 1 \mathrm{H}), 7.69-7.71(\mathrm{~m}, 2 \mathrm{H}), 7.59-7.61(\mathrm{~m}, 2 \mathrm{H}), 3.95(\mathrm{~s}, 3 \mathrm{H})$ ppm. ${ }^{13} \mathrm{C}$ NMR $\left(100.61 \mathrm{MHz}, \mathrm{CDCl}_{3}\right): \delta 183.0,151.8,134.3,131.6,130.2,130.0,123.7,115.4,36.4 \mathrm{ppm} .{ }^{15} \mathrm{~N}$ NMR (40.56 MHz, CDCl 3 ): $\delta$-74.6, -177.1 ppm. MS (El, $70 \mathrm{eV}): \mathrm{m} / \mathrm{z}$ (\%) 298 (70, [M+]), 219 (53), 109 (29), 76 (42), 50 (18). Anal. calcd. for $\mathrm{C}_{11} \mathrm{H}_{8} \mathrm{BrClN}_{2} \mathrm{O}: \mathrm{C}, 44.11 ; \mathrm{H}, 2.69 ; \mathrm{Br}, 26.67 ; \mathrm{Cl}, 11.84 ; \mathrm{N}, 9.35$. Found \%: $\mathrm{C}, 44.07 ; \mathrm{H}$, $2.68 ; \mathrm{Cl}, 11.88 ; \mathrm{N}, 9.32$. 
5-Chloro-3-(4-fluorophenyl)-1-methyl-1H-pyrazole-4-carbaldehyde (2k). The general procedure was followed using 5-chloro-3-(4-fluorophenyl)-1-methyl-1H-pyrazole (1k, $2 \mathrm{mmol})$, reaction time: $10 \mathrm{~h}, 253 \mathrm{mg}$ (53\%), peach powder, m.p. 226-227 ${ }^{\circ} \mathrm{C}$; $R_{f} 0.37$ (diethyl ether/hexane, 1:1 v/v). IR (film): 3076, 2945, 1684, 1499, 1446, $778 \mathrm{~cm}^{-1} .{ }^{1} \mathrm{H}$ NMR $\left(400.13 \mathrm{MHz}, \mathrm{CDCl}_{3}\right): \delta 9.93(\mathrm{~s}, 1 \mathrm{H}), 7.78-7.81(\mathrm{~m}, 2 \mathrm{H}), 7.13-7.17(\mathrm{~m}, 2 \mathrm{H}), 3.95(\mathrm{~s}, 3 \mathrm{H}$, $\left.\mathrm{CH}_{3}\right)$ ppm. ${ }^{13} \mathrm{C}$ NMR $\left(100.61 \mathrm{MHz}, \mathrm{CDCl}_{3}\right): \delta 183.0,163.4\left(\mathrm{~d},{ }^{1} J_{\mathrm{C}, \mathrm{F}} 249.3 \mathrm{~Hz}\right), 151.9,134.1,130.5\left(\mathrm{~d},{ }^{3} \mathrm{~J}_{\mathrm{C}, \mathrm{F}} 8.4 \mathrm{~Hz}\right)$, $127.1\left(\mathrm{~d},{ }^{4} J_{\mathrm{C}, \mathrm{F}} 3.2 \mathrm{~Hz}\right), 115.4\left(\mathrm{~d},{ }^{2} \mathrm{~J}_{\mathrm{C}, \mathrm{F}} 21.6 \mathrm{~Hz}\right), 115.2,36.2 \mathrm{ppm} .{ }^{15} \mathrm{~N} \mathrm{NMR}\left(40.56 \mathrm{MHz}, \mathrm{CDCl}_{3}\right): \delta-75.4,-177.4$ ppm. MS (El, $70 \mathrm{eV}): \mathrm{m} / \mathrm{z}$ (\%) 238 (100, [M+]), 203 (27), 175 (20), 156 (41), 131 (18), 107 (40), 95 (32), 76 (54). Anal. calcd. for $\mathrm{C}_{11} \mathrm{H}_{8} \mathrm{ClFN}_{2} \mathrm{O}$ : C, 55.36; H, 3.38; Cl, 14.86; F, 7.96, N, 11.74. Found \%: $\mathrm{C}, 55.45 ; \mathrm{H}, 3.39 ; \mathrm{Cl}, 14.80$; N, 11.75 .

5-Chloro-1-methyl-3-(4-nitrophenyl)-1H-pyrazole-4-carbaldehyde (2l). The general procedure was followed using 5-chloro-1-methyl-3-(4-nitrophenyl)-1H-pyrazole (1m, $2 \mathrm{mmol})$, reaction time: $14 \mathrm{~h}, 244 \mathrm{mg}$ (46\%), beige powder, mp 171-172 ${ }^{\circ} \mathrm{C}$; $R_{f} 0.23$ (diethyl ether/hexane, 1:1 v/v). IR (film): 3106, 3080, 2922, 2850, 1688, 1519, 1345, $778 \mathrm{~cm}^{-1} .{ }^{1} \mathrm{H}$ NMR $\left(400.13 \mathrm{MHz}, \mathrm{CDCl}_{3}\right): \delta 9.97(\mathrm{~s}, 1 \mathrm{H}), 8.29-8.31(\mathrm{~m}, 2 \mathrm{H}), 8.08-8.10(\mathrm{~m}, 2 \mathrm{H}), 3.99(\mathrm{~s}, 3 \mathrm{H})$ ppm. ${ }^{13} \mathrm{C}$ NMR $\left(100.61 \mathrm{MHz}, \mathrm{CDCl}_{3}\right): \delta 182.6,149.9,148.2,137.4,135.7,129.5,123.6,115.8,36.6 \mathrm{ppm} .{ }^{15} \mathrm{~N}$ NMR (40.56 MHz, CDCl $): \delta$-8.1, -69.9, -176.3 ppm. MS (El, $70 \mathrm{eV}): \mathrm{m} / \mathrm{z}(\%) 265$ (100, [M+]), 248 (16), 218 (33), 107 (29), 76 (27), 50 (15). Anal. calcd. for $\mathrm{C}_{11} \mathrm{H}_{8} \mathrm{ClN}_{3} \mathrm{O}_{3}$ : $\mathrm{C}, 49.73 ; \mathrm{H}, 3.04 ; \mathrm{Cl}, 13.35 ; \mathrm{N}, 15.82$. Found \%: $\mathrm{C}, 49.81$; $\mathrm{H}, 3.03 ; \mathrm{Cl}, 13.30 ; \mathrm{N}, 15.77$.

5-Chloro-1,3-dimethyl-1H-pyrazole-4-carbaldehyde (2m). The general procedure was followed using 5-chloro1,3-dimethyl-1H-pyrazole (1l, $2 \mathrm{mmol}$ ), reaction time: $1 \mathrm{~h}, 209 \mathrm{mg}(66 \%)$, orange needles, $\mathrm{mp} 67-68^{\circ} \mathrm{C}, R_{f} 0.38$ (diethyl ether/hexane, 2:1 v/v). IR (film): 2937, 1682, 1528, 1476, $771 \mathrm{~cm}^{-1} .{ }^{1} \mathrm{H} \mathrm{NMR}\left(400.13 \mathrm{MHz}, \mathrm{CDCl}_{3}\right): \delta$ $9.95(\mathrm{~s}, 1 \mathrm{H}), 3.81(\mathrm{~s}, 3 \mathrm{H}), 2.44(\mathrm{~s}, 3 \mathrm{H}) \mathrm{ppm} .{ }^{13} \mathrm{C}$ NMR $\left(100.61 \mathrm{MHz}, \mathrm{CDCl}_{3}\right): \delta 183.3,150.8,133.6,116.2,35.8$, 13.6 ppm. ${ }^{15} \mathrm{~N} \mathrm{NMR}\left(40.56 \mathrm{MHz}^{\mathrm{CDCl}} 3\right): \delta-77.0,-180.5 \mathrm{ppm}$. MS (El, $\left.70 \mathrm{eV}\right): \mathrm{m} / \mathrm{z}(\%) 158(54,[\mathrm{M}+]), 157(100)$. Anal. calcd. for $\mathrm{C}_{6} \mathrm{H}_{7} \mathrm{CIN} 2 \mathrm{O}: \mathrm{C}, 45.44 ; \mathrm{H}, 4.45 ; \mathrm{Cl}, 22.36 ; \mathrm{N}, 17.66$. Found \%: C, 45.29; $\mathrm{H}, 4.44 ; \mathrm{Cl}, 22.41 ; \mathrm{N}, 17.61$. 5-Chloro-3-(chloromethyl)-1-methyl-1H-pyrazole-4-carbaldehyde (2n). The general procedure was followed using 5-chloro-3-(chloromethyl)-1-methyl-1H-pyrazole (1n, $2 \mathrm{mmol}$ ), reaction time: $1 \mathrm{~h}, 228 \mathrm{mg}$ (59\%), white powder, mp 105-106 ${ }^{\circ} \mathrm{C}, R_{f} 0.20$ (diethyl ether/hexane, 1:1 v/v). IR (film): 2927, 2858, 1671, 1517, 1482,769 $\mathrm{cm}^{-1} .{ }^{1} \mathrm{H}$ NMR $\left(400.13 \mathrm{MHz}, \mathrm{CDCl}_{3}\right): \delta 9.90(\mathrm{~s}, 1 \mathrm{H}), 4.78(\mathrm{~s}, 2 \mathrm{H}), 3.89(\mathrm{~s}, 3 \mathrm{H}) \mathrm{ppm} .{ }^{13} \mathrm{C} \mathrm{NMR}\left(100.61 \mathrm{MHz}, \mathrm{CDCl}_{3}\right):$ $\delta$ 182.5, 149.4, 134.1, 115.7, 36.9, 36.3 ppm. $\left.{ }^{15} \mathrm{~N} \mathrm{NMR} \mathrm{(40.56} \mathrm{MHz,} \mathrm{CDCl}_{3}\right): \delta-70.1,-178.4 \mathrm{ppm} . \mathrm{MS}$ (El, $\left.70 \mathrm{eV}\right)$ : m/z (\%) 193 (43, [M+]), 192 (80), 191 (59), 157 (100), 156 (50), 76 (41). Anal. calcd. for $\mathrm{C}_{6} \mathrm{H}_{6} \mathrm{Cl}_{2} \mathrm{~N}_{2} \mathrm{O}: \mathrm{C}, 37.33 ; \mathrm{H}$, 3.13; $\mathrm{Cl}, 36.73 ; \mathrm{N}, 14.51$. Found \%: C, 37.20; $\mathrm{H}, 3.14 ; \mathrm{Cl}, 36.75 ; \mathrm{N}, 14.49$.

5-Chloro-3-(1-chloroethyl)-1-methyl-1H-pyrazole-4-carbaldehyde (2o). The general procedure was followed using 5-chloro-3-(1-chloroethyl)-1-methyl-1H-pyrazole (10, $2 \mathrm{mmol})$, reaction time: $2 \mathrm{~h}, 17 \mathrm{mg}(4 \%) ; R_{f} 0.27$ (diethyl ether/hexane, 1:2 v/v). ${ }^{1} \mathrm{H}$ NMR (400.13 MHz, $\left.\mathrm{CDCl}_{3}\right): \delta 9.92(\mathrm{~s}, 1 \mathrm{H}), 5.58(\mathrm{q}, J 6.9 \mathrm{~Hz}, 1 \mathrm{H}), 3.88(\mathrm{~s}, 3 \mathrm{H})$, 1.88 (d, J $6.9 \mathrm{~Hz}, 3 \mathrm{H}$ ) ppm. ${ }^{13} \mathrm{C}$ NMR $\left(100.61 \mathrm{MHz}, \mathrm{CDCl}_{3}\right): \delta 182.6,150.0,134.2,114.8,49.4,36.3,23.1 \mathrm{ppm}$. ${ }^{15} \mathrm{~N} \mathrm{NMR}\left(40.56 \mathrm{MHz} \mathrm{CDCl}_{3}\right): \delta-72.3,-179.4 \mathrm{ppm}$.

(5-chloro-1-phenyl-3-propyl-1H-pyrazol-4-yl)methanol (3). The general procedure was followed using 5chloro-1-phenyl-3-propyl-1H-pyrazole (1f, $2 \mathrm{mmol}$ ), reaction time: $14 \mathrm{~h}, 30 \mathrm{mg}$, (6\%), yellow oil, $R_{f} 0.40$ (diethyl ether/hexane, 1:8 v/v). IR (film): 3062, 2960, 2929, 2869, 1551, 1502, 1464, 1423, 761, $693 \mathrm{~cm}^{-1} .{ }^{1} \mathrm{H} \mathrm{NMR}$ (400.13 MHz, $\left.\mathrm{CDCl}_{3}\right): \delta$ 7.54-7.56 $(\mathrm{m}, 1 \mathrm{H}), 7.46-7.50(\mathrm{~m}, 2 \mathrm{H}), 7.39-7.42(\mathrm{~m}, 2 \mathrm{H}), 4.57(\mathrm{~s}, 2 \mathrm{H}), 2.71(\mathrm{t}, J 7.8 \mathrm{~Hz}$, $2 \mathrm{H}), 1.75-1.81(\mathrm{~m}, 2 \mathrm{H}), 1.04(\mathrm{t}, J 7.3 \mathrm{~Hz}, 3 \mathrm{H}) \mathrm{ppm} .{ }^{13} \mathrm{C} \mathrm{NMR}\left(100.61 \mathrm{MHz}, \mathrm{CDCl}_{3}\right): \delta 153.1,138.2,129.0,128.2$, 127.0, 124.9, 114.2, 29.1, 22.3, 16.1, 13.8 ppm. ${ }^{15} \mathrm{~N}$ NMR (40.56 MHz, CDCl$): \delta-76.0,-169.1$ ppm. MS (El, 70 eV): $m / z$ (\%) 233 [100], 205 [64], 77 [84], 51 [66]. Anal. calcd. for $\mathrm{C}_{13} \mathrm{H}_{15} \mathrm{ClN} 2 \mathrm{O}: \mathrm{C}, 62.28 ; \mathrm{H}, 6.03 ; \mathrm{Cl}, 14.14 ; \mathrm{N}$, 11.17. Found \%: C, 62.18; $\mathrm{H}, 6.02 ; \mathrm{Cl}, 14.18 ; \mathrm{N}, 11.21$. 
5-chloro-3-ethenyl-1-methyl-1H-pyrazole-4-carbaldehyde (4). The general procedure was followed using 5chloro-3-(1-chloroethyl)-1-methyl-1H-pyrazole (10, $2 \mathrm{mmol}$ ), reaction time: $2 \mathrm{~h}, 24 \mathrm{mg}$ (7\%), $R_{f} 0.27$ (diethyl ether/hexane, 1:2 v/v). ${ }^{1} \mathrm{H}$ NMR $\left(400.13 \mathrm{MHz}, \mathrm{CDCl}_{3}\right): \delta 9.90(\mathrm{~s}, 1 \mathrm{H}), 7.04(\mathrm{dd}, J 11.3,17.7 \mathrm{~Hz}, 1 \mathrm{H}), 6.22(\mathrm{dd}, J$ 17.7, $1.0 \mathrm{~Hz}, 1 \mathrm{H}), 5.50$ (dd, J 11.3, $1.0 \mathrm{~Hz}, 1 \mathrm{H}), 3.88(\mathrm{~s}, 3 \mathrm{H}) \mathrm{ppm} .{ }^{13} \mathrm{C} \mathrm{NMR}(100.61 \mathrm{MHz}, \mathrm{CDCl} 3): \delta 183.0,150.0$, 133.9, 126.3, 119.8, 115.4, 36.2 ppm. $\left.{ }^{15} \mathrm{~N} \mathrm{NMR} \mathrm{(40.56} \mathrm{MHz}^{\mathrm{CDCl}} 3\right): \delta-78.8,-179.4 \mathrm{ppm}$.

(2E)-3-(5-chloro-1-methyl-1H-pyrazol-3-yl)prop-2-enal (5). The general procedure was followed using 5chloro-3-(1-chloroethyl)-1-methyl-1H-pyrazole (10, $2 \mathrm{mmol}$ ), reaction time: $2 \mathrm{~h}, 246 \mathrm{mg}$ (72\%), white powder, $\mathrm{mp} 68-70^{\circ} \mathrm{C}, R_{f} 0.15$ (diethyl ether/hexane, 1:2 v/v). IR (film): 3134, 2851, 2827, 1672, 1633, 1504, 974,786 $\mathrm{cm}^{-1} .{ }^{1} \mathrm{H}$ NMR $\left(400.13 \mathrm{MHz}, \mathrm{CDCl}_{3}\right): \delta 9.87(\mathrm{~d}, J 7.8 \mathrm{~Hz}, 1 \mathrm{H}), 7.37(\mathrm{~d}, J 15.9 \mathrm{~Hz}, 1 \mathrm{H}), 6.59(\mathrm{dd}, J 7.8,15.9 \mathrm{~Hz}, 1 \mathrm{H})$, 6.52(s, 1H), $3.90(\mathrm{~s}, 3 \mathrm{H}) \mathrm{ppm} .{ }^{13} \mathrm{C}$ NMR $\left(100.61 \mathrm{MHz}, \mathrm{CDCl}_{3}\right): \delta 193.4,146.9,143.4,129.5,129.2,104.0,36.6$ ppm. ${ }^{15} \mathrm{~N} N M R\left(40.56 \mathrm{MHz} \mathrm{CDCl}_{3}\right): \delta$-66.1, -175.6 ppm. MS (El, $\left.70 \mathrm{eV}\right) \mathrm{m} / z$ \%: 170 (20, [M+]), $142(100), 141$ (53), 107 (22), 81 (32). Anal. calcd. for $\mathrm{C}_{7} \mathrm{H}_{7} \mathrm{ClN} 2 \mathrm{O}: \mathrm{C}, 49.28 ; \mathrm{H}, 4.14 ; \mathrm{Cl}, 20.78 ; \mathrm{N}, 16.42$. Found \%: $\mathrm{C}, 49.16 ; \mathrm{H}$, 4.16; Cl, 20.73; N, 16.47 .

5(3)-Chloro-3(5)-propyl-1H-pyrazole (6). The general procedure was followed using 1-tert-butyl-5-chloro-3propyl-1H-pyrazole (1p, $2 \mathrm{mmol}$ ), reaction time: $2 \mathrm{~h}, 252 \mathrm{mg}(87 \%)$, yellow oil, $R_{f} 0.20$ (diethyl ether/hexane, $1: 1 \mathrm{v} / \mathrm{v})$. IR (film): 3186, 3141, 2965, 2871, 1576, 1462, 1368, $798 \mathrm{~cm}^{-1} .{ }^{1} \mathrm{H} \mathrm{NMR}\left(400.13 \mathrm{MHz}, \mathrm{CDCl}_{3}\right): \delta 6.00(\mathrm{~s}$, $1 \mathrm{H}), 2.69(\mathrm{t}, 2 \mathrm{H}, \quad J 7.6 \mathrm{~Hz}), 1.67(\mathrm{~m}, 2 \mathrm{H}), 0.98(\mathrm{t}, 3 \mathrm{H}, J 7.3 \mathrm{~Hz}) \mathrm{ppm} .{ }^{13} \mathrm{C} \mathrm{NMR}(100.61 \mathrm{MHz}, \mathrm{CDCl} 3): \delta 146.6$, 139.7, 102.7, 27.8, 22.4, 13.6 ppm. ${ }^{15} \mathrm{~N}$ NMR (40.56 MHz): $\delta-101.3,-178.3 \mathrm{ppm} . \mathrm{MS}$ (El, $\left.70 \mathrm{eV}\right): \mathrm{m} / \mathrm{z}(\%) 144$ (47, [M+]), 116 (100), 115 (92). Anal. calcd. for $\mathrm{C}_{6} \mathrm{H}_{9} \mathrm{ClN}_{2}$ : C, 49.84; $\mathrm{H}, 6.27 ; \mathrm{Cl}, 24.52 ; \mathrm{N}, 19.37$. Found \%: C, 50.00; $\mathrm{H}, 6.28 ; \mathrm{Cl}, 24.46 ; \mathrm{N}, 19.30$.

\section{Acknowledgements}

The spectral and analytical data were obtained using the equipment of the Baykal Analytical Center for Collective Use SB RAS.

\section{Supplementary Material}

Copies of spectra of compounds are provided in the supplementary material file available on the Publisher's web site.

\section{References}

1. Ansari, A.; Ali, A.; Asif, M.; Shamsuzzaman. New J. Chem. 2017, 41, 16. http://dx.doi.org/10.1039/c6nj03181a

2. Ardiansah, B. Asian J. Pharm. Clin. Res. 2017, 10, 45. http://dx.doi.org/10.22159/ajpcr.2017.v10i12.22065

3. Kumar V.; Sareen, V.; Khatri, V.; Sareen, S. Int. J. Applied Res. 2016, 2, 461.

4. Vicentini, C.B.; Romagnoli, C.; Andreotti, E.; Mares, D. J. Agric. Food. Chem. 2007, 55, 10331. http://dx.doi.org/10.1021/jf072077d 
5. Giornal, F.; Pazenok, S.; Rodefeld, L.; Lui, N.; Vors, J.-P.; Leroux, F.R. J. Fluor. Chem. 2013, $152,2$. http://dx.doi.org/10.1016/j.jfluchem.2012.11.008

6. Lei. P.; Zhang, X.; Xu, Y.; Xu, G.; Liu, X.; Yang, X.; Zhang, X.; Ling, Yun. Chem. Cent. J. 2016, $10,40$. http://dx.doi.org/10.1186/s13065-016-0186-8

7. Halcrow, M.A. Dalton Trans. 2009, 2059.

http://dx.doi.org/10.1039/b815577a

8. Potapov, A.S.; Chernova, N.P.; Ogorodnikov, V.D.; Petrenko, T.V.; Khlebnikov, A.I. Beilstein J. Org. Chem. 2011, 7, 1526.

http://dx.doi.org/10.3762/bjoc.7.179

9. Bianchi, L.; Carloni-Garaventa, A.; Maccagno, M.; Pani, M.; Petrillo, G.; Scapolla, C.; Tavani, C. Tetrahedron 2015, 71, 7550.

http://dx.doi.org/10.1016/j.tet.2015.08.014

10. Taylor, R.D.; MacCoss, M.; Lawson, A.D.G. J. Med. Chem. 2014, 57, 5845. http://dx.doi.org/10.1021/jm4017625

11. Abdel-Wahab, $\quad$ B.F.; Khidre, R.E.; Farahat, A.A. Arkivoc 2011, (i), 196. http://dx.doi.org/10.3998/ark.5550190.0012.103

12. Schmidt, A.; Dreger, A. Curr. Org. Chem. 2011, 15, 1423.

http://dx.doi.org/10.2174/138527211795378263

13. Janin Y.L. Chem. Rev. 2012, 112, 3924.

http://dx.doi.org/10.1021/cr200427q

14. Fuestro, S.; Sanchez-Rosello, M.; Barrio, P.; Simon-Fuentes, A. Chem. Rev. 2011, $111,6984$. http://dx.doi.org/10.1021/cr2000459

15. Bourrain, S.; Ridgill, M.; Collins, I. Synlett 2004, 5, 795.

http://dx.doi.org/10.1055/s-2004-820020

16. Rodriguez-Franco, M.I; Dorronsoro, I.; Martinez, A. Synthesis 2001, 11, 1711.

http://dx.doi.org/10.1055/s-2001-16768

17. Ovcharenko, V.; Fokin, S.; Chubakova, E.; Romanenko, G.; Bogomyakov, A.; Dobrokhotova, Zh.; Lukzen, N.; Morozov, V.; Petrova, M.; Petrova, M.; Zueva, E.; Rozentsveig, I.; Rudyakova, E.; Levkovskaya, G.; Sagdeev, R. Inorg. Chem. 2016, 55, 5853.

http://dx.doi.org/10.1021/acs.inorgchem.6b00140

18. Papernaya, L.K.; Shatrova, A.A.; Albanov, A.I.; Levkovskaya, G.G.; Rozentsveig, I.B. Arkivoc 2016, (v), 142. http://dx.doi.org/10.3998/ark.5550190.p009.709

19. Abu-Hashem A.A.; Gouda M.A.; Badria F.A. Eur. J. Med. Chem. 2010, 45, 1976. https://doi.org/10.1016/j.ejmech.2010.01.042

20. Gouda M. A.; Abu-Hashem A.A.; Saad, H.H.; Elattar K.M. Res. Chem. Intermed. 2016, $42,2119$. https://doi.org/10.1007/s11164-015-2139-6

21. Paul, S.; Gupta, M.; Gupta, R. Synlett. 2000, 8, 1115.

https://doi.org/10.1055/s-2000-6747

22. Sumathy, A.; Gowrishankar, N. L.; Krishnan, A; Prakash, M; Muhsin, T.; Naseena, U.; Poorni ma, G. World J. of Pharmacy and Pharmaceutical Sciences 2018, 7, 574.

https://doi.org/10.20959/wjpps20185-11603

23. Butler, D.E.; DeWald, H.A. J. Org. Chem. 1971, 36, 2542.

https://doi.org/10.1021/jo00816a037

24. Buchi, J.; Ursprung, R.; Lauener, G. Helv. Chim. Acta 1949, 32, 984. 
https://doi.org/10.1002/hlca.19490320347

25. Despotopoulou, C.; Klier, L.; Knochel, P. Org. Lett. 2009, 11, 3326.

https://doi.org/10.1021/ol901208d

26. Hamatake, R.K.; Chen, H.; Raney, A.; Allan, M.; Lang, S. Patent WO 2006033995, 2006.

27. Bozhenkov, G.V.; Savosik, V.A.; Larina, L.I.; Klyba, L.V.; Zhanchipova, E.R.; Mirskova, A.N.; Levkovskaya, G.G. Russ. J. Org. Chem. 2008, 44, 1014.

https://doi.org/10.1134/S1070428008070129

28. Bozhenkov, G.V.; Savosik, V.A.; Larina, L.I.; Klyba, L.V.; Zhanchipova, E.R.; Mirskova, A.N.; Levkovskaya, G.G. Russ. J. Org. Chem. 2008, 44, 1194.

https://doi.org/10.1134/S1070428008080150

29. Rudyakova, E.V.; Savosik, V.A.; Evstaf'eva, I.T.; Kondrashov, E.V.; Levkovskaya, G.G. Russ. J. Org. Chem. 2009, 45, 705.

https://doi.org/10.1134/S1070428009050108

30. Levkovskaya, G.G.; Kobelevskaya, V.A.; Rudyakova, E.V.; Khanh, Q. Ha.; Samultsev, D.O.; Rozentsveig, I. B. Tetrahedron 2011, 67, 1844.

https://doi.org/10.1016/j.tet.2011.01.028

31. Levkovskaya, G.G.; Atavin, A.S.; Mirskova, A.N. Zh. Org. Khim. 1973, 9, 318. Chem. Abstr. 1973, 78, 159137g.

32. Mirskova, A.N.; Levkovskaya, G.G.; Kalikhman, I.D.; Voronkov, M.G. Zh. Org. Khim. 1979, 15, 2301. Chem. Abstr. 1980, 92, 128792b.

33. Levkovskaya, G.G.; Mirskova, A.N.; Kalikhman, I.D.; Voronkov, M.G. Zh. Org. Khim. 1984, 21, 634. Chem. Abstr. 1984, 101, 90823b.

34. Levkovskaya, G.G.; Bozhenkov, G.V.; Larina, L.I.; Evstaf'eva, I.T.; Mirskova, A.N. Russ. J. Org. Chem. 2001, 37, 644.

https://doi.org/10.1023/A:1012483314133

35. Barreiro, E.J.; Camara, C.A.; Verli, H.; Brazil-Mas, L.; Castro, N.J.; Cintra, W.M.; Aracava, Y.; Rodrigues, C.R.; Fraga, C.A.M. J. Med. Chem. 2003, 46, 1144.

https://doi.org/10.1021/jm020391n

36. Popov, A.V.; Kobelevskaya, V.A.; Larina, L.I.; Levkovskaya, G.G. Mendeleev Commun. 2017, $27,178$. https://doi.org/10.1016/i.mencom.2017.03.024

37. Badalyan, K.S.; Akopyan, A.E.; Attaryan, H.S.; Astratyan, G.V. Russ. J. Gen. Chem. 2014, $84,793$. https://doi.org/10.1134/S1070363214040331

38. Kamigata, N.; Udodaira, K.; Yoshikawa, M.; Shimizu, T. J. Organomet. Chem. 1998, 552, 39.

https://doi.org/10.1016/S0022-328X(97)00497-X 